\title{
Stability of antiphase line defects in nanometer-sized boron-nitride cones
}

\author{
Sérgio Azevedo ${ }^{1,2}$, Mário S. C. Mazzoni ${ }^{1}$, R. W. Nunes ${ }^{1}$, and H. Chacham* \\ 1 Departamento de Física, ICEX, Universidade Federal de Minas Gerais, \\ CP 702, 30123-970, Belo Horizonte, MG, Brazil. and \\ 2 Departamento de Física, Universidade Estadual de Feira de Santana, \\ Km 3 BR-116, 44031-460, Feira de Santana, BA, Brazil.
}

(Dated: June 28, 2018)

\begin{abstract}
We investigate the stability of boron nitride conical sheets of nanometer size, using first-principles calculations. Our results indicate that cones with an antiphase boundary (a line defect that contains either B-B or N-N bonds) can be more stable than those without one. We also find that doping the antiphase boundaries with carbon can enhance their stability, leading also to the appearance of localized states in the bandgap. Among the structures we considered, the one with the smallest formation energy is a cone with a carbon-modified antiphase boundary that presents a spin splitting of $\sim 0.5 \mathrm{eV}$ at the Fermi level.

PACS numbers: 71.20.Tx, 71.15.Mb, 71.24.+q
\end{abstract}

\section{INTRODUCTION}

Boron nitride (BN) and carbon can be found in similar structures, from the diamond and graphite bulk forms, to nanostructures such as nanotubes,$\frac{1,2.3 .4}{}$ fullerenes 5.6 and conical sheets ${ }^{7.8}$ However, unlike carbon, BN structures can contain three types of covalent bonds, namely $\mathrm{B}-\mathrm{N}, \mathrm{N}-\mathrm{N}$, and B-B ${ }^{9.10}$ This leads to a qualitative difference between $\mathrm{BN}$ and carbon in the formation of the graphite-derived curved surfaces that are found in nanostructures such as fullerenes and nanotube caps. In carbon, energy minimization leads to structures made of $60^{\circ}$ disclinations (conical sheets) ${ }^{11}$ with isolated pentagons at the apex. Carbon fullerenes are built from such structures. In BN, fullerene-like structures are formed from $120^{\circ}$ disclinations. ${ }^{6}$ These $120^{\circ} \mathrm{BN}$ disclinations are usually modeled as having a four-membered ring at the apex, $\stackrel{6,12.13}{b u t}$ theoretical calculations indicate that non-stoichiometric BN fullerenes, consisting of pentagon pairs replacing the four-membered rings, may be energetically favored, depending on the stoichiometric condition of growth 14.15

A possible reason for the existence of $120^{\circ}$ disclinations in $\mathrm{BN}$ nanostructures, instead of the $60^{\circ}$ ones that are found in carbon, is the existence of antiphase boundaries (APB) in BN disclinations with an odd multiple of $60^{\circ} \underline{16.17}$ The common perception is that these APB's, which necessarily contain a line defect of non-BN bonds, should present an energy cost that surpasses the lower elastic-energy cost of a disclination of smaller angle. However, such a "no-wrong-bond" rule 13 has been challenged by at least two experiments. In their work, Bourgeois et al $l^{8}$ have shown the existence of $\mathrm{BN}$ conical nanostructures with a disclination angle of $300^{\circ}$, demonstrating the existence of APB's. Another example in the literature is the observation of disclinations in $\mathrm{BN}$ nanotube junctions, 18 in experiments whose interpretation is also compatible with the existence of an APB.

In the present work, we investigate the relative stability of nanometer-sized $\mathrm{BN}$ cones with and without
APB's, for N-rich and B-rich environments. Our firstprinciples results indicate that, for these two limiting stoichiometric situations, structures with an APB can be more stable than APB-free structures, consisting only of $\mathrm{B}-\mathrm{N}$ bonds. We also find structures involving full carbon incorporation at the APB to be among the most stable, under both environments.

The paper is organized as follows: in Sec. I we discuss the $a b$ initio methodology for the computation of the total energies and the definition of chemical potentials for the computation of the zero-Kelvin grand potentials; in Sec. III we discuss our model for the formation energy as a sum of antiphase boundary and elastic energies; in $\mathrm{Sec}$.IV we discuss our first principles results for the relative stability of the various cones and some features of the electronic states of the most stable structures; and finally, in Sec. $\mathbb{\nabla}$ we present some discussion of scaling to larger structures than considered in this work and conclusions.

\section{METHODOLOGY}

\section{A. Total energy calculations}

Our calculations are based on the density functional theory ${ }^{19}$ as implemented in the SIESTA program ${ }^{20}$ We make use of norm-conserving Troullier-Martins pseudopotentials ${ }^{21}$ in the Kleinman-Bylander factorized form,, 22 and a double- $\zeta$ basis set composed of numerical atomic orbitals of finite range. Polarization orbitals are included for nitrogen, boron and carbon atoms, and we use the generalized gradient approximation $(\mathrm{GGA})^{23}$ for the exchange-correlation potential. All the geometries are fully relaxed, with residual forces smaller than 0.1 $\mathrm{eV} / \AA$. 


\section{B. Zero-Kelvin grand potentials}

The comparison of BN clusters with different number of atoms is a difficult problem. Here, we propose a zero-temperature thermodynamic approach based on the prior determination of chemical potentials to address this issue. The robustness of the approach will be established by comparison with results obtained with different chemical potentials, which represent different experimental conditions, and with similar calculations in the literature $14,24,25,26,27$

In order to address the energetics of stoichiometric and nonstoichiometric cones, we must distinguish the different techniques that can be employed in their synthesis. In each case, we introduce suitable theoretical chemical potentials for nitrogen $\left(\mu_{N}\right)$ and boron $\left(\mu_{B}\right)$. For the case in which the cones are obtained by laser ablation or arc-discharge from hexagonal BN, we can have either a nitrogen- or a boron-rich environment, depending on the specific atomic reservoir employed. In the N-rich environment, $\mu_{N}$ is obtained from nitrogen in the gas phase, while a metallic $\alpha$-B phase is used as the reservoir for the B-rich environment. In both cases, $\mu_{N}$ and $\mu_{B}$ are linked by the thermodynamic constraint

$$
\mu_{N}+\mu_{B}=\mu_{B N}^{\text {layer }}
$$

where $\mu_{B N}^{\text {layer }}$ is the chemical potential per BN pair in the infinite, planar BN sheet. This constraint corresponds to situations in which the nanostructures are formed in equilibrium with BN layers. Since we are dealing with finite clusters, we use hydrogen atoms to saturate the dangling bonds at the edges. To take these additional $\mathrm{H}-\mathrm{B}$ and $\mathrm{H}-\mathrm{N}$ bonds into account, we introduce the respective chemical potentials, $\mu_{H B}$ and $\mu_{H N}$, and write the formation energy of the cones as

$$
\begin{aligned}
E_{\text {form }}= & E_{t o t}-n_{B} \mu_{B}-n_{N} \mu_{N}-n_{H N} \mu_{H N} \\
& -n_{H B} \mu_{H B},
\end{aligned}
$$

where $E_{\text {tot }}$ is the calculated total energy of the cluster, $n_{B}$ and $n_{N}$ are the number of $\mathrm{B}$ and $\mathrm{N}$ atoms, and $n_{H N}$ and $n_{H B}$ are the number of $\mathrm{H}-\mathrm{B}$ and $\mathrm{H}-\mathrm{N}$ bonds, respectively. The formation energy, as defined in Eq. 2] represents the zero-Kelvin grand potential which is the proper thermodynamical potential for the comparison of the relative stability of structures with different number of atoms.

A first constraint on the hydrogen chemical potentials is imposed by using a finite planar sheet of boron nitride as reference, and ascribing a null value to its formation energy. This allows us to write its total energy as

$$
E_{T}^{\text {sheet }}=n_{B N} \mu_{B N}^{\text {layer }}+n_{H B} \mu_{H B}+n_{H N} \mu_{H N},
$$

where $n_{B N}$ is the number of BN pairs.

The planar reference sheet is centered at a sixfold ring and has $C_{3 v}$ symmetry. Therefore, $n_{H B}=n_{H N}$ and the preceding equation can be rewritten as

$$
E_{T}^{\text {sheet }}=n_{B N} \mu_{B N}^{\text {layer }}+n_{H} \mu_{H},
$$

where we have defined $\mu_{H}=\left(\mu_{B H}+\mu_{N H}\right) / 2$. Using the total energy calculations for the infinite layer and the finite sheet, we find $\mu_{H}=-15.46 \mathrm{eV}$.

From the definition of $\mu_{H}$, we obtain $\mu_{H B}\left(\mu_{H N}\right)$ once $\mu_{H N}\left(\mu_{H B}\right)$ is known. The values of one of these parameters can be determined by choosing a convenient reservoir. In our calculations we use the ammonia molecule $\left(\mathrm{NH}_{3}\right)$ as a reservoir, obtaining $\mu_{H N}=-16.16 \mathrm{eV}$ and $\mu_{H B}=-14.76 \mathrm{eV}$ in the N-rich environment, and $\mu_{H N}=$ $-15.23 \mathrm{eV}$ and $\mu_{H B}=-15.69 \mathrm{eV}$ in the B-rich environment. The consistency of this methodology was tested by determining $\mu_{H B}$ using the $\mathrm{BH}_{3}$ molecule as a reservoir, and then calculating $\mu_{H N}$ from $\mu_{H}$. The calculated formation energies in this case are within $0.01 \mathrm{eV} /$ atom of those from the $\mathrm{NH}_{3}$ reservoir. For the structures containing carbon, the chemical potentials for the carbon atom $\left(\mu_{C}\right)$ and the carbon-hydrogen bonds $\left(\mu_{C H}\right)$ are obtained from total energy calculations for bulk graphene and for a finite carbon sheet saturated by hydrogen atoms, respectively.

\section{FORMATION ENERGY: ANTIPHASE-BOUNDARY AND ELASTIC CONTRIBUTIONS}

The physical mechanism that defines whether structures having APB's may be as stable as their APB-free counterparts is the competition between the elastic energy associated with the cone formation and the energetic cost of forming an APB. Hence, we write the cone formation energies, calculated with the above procedure, as the sum of two contributions. The first is the elastic energy required to fold a finite sheet into a cone, which we describe by a continuum model. The second represents the APB chemical energetic cost associated with the non-BN bonds.

The basic assumption of the continuum model is that the elastic energy stored in a differential element of area at the surface of the cone is proportional to the square of the surface mean curvature in that region. Within this model, the elastic energy of the cone is written

$$
E_{e l}=K\left(\frac{\cos ^{2} \alpha}{\sin \alpha}\right) \ln \left(\frac{R}{R_{c}}\right)+E_{t i p}\left(R_{c}\right),
$$

where $2 \alpha$ is the apex angle, $R$ is the slant height, $R_{c}$ is a cutoff length introduced to remove the singularity at the tip (linear elasticity breaks down in the neighborhood of the tip), and $E_{t i p}\left(R_{c}\right)$ is the associated tip energy. The expression

$$
\sin (\alpha)=1-\frac{\beta}{2 \pi}=1-\frac{N}{6}
$$

relates the apex angle to the disclination angle $\beta=N \times$ $60^{\circ}$. 
The validity of the continuum model was first tested for the case of carbon, for which there is no APB. We found that the first-principles total energy calculations for the graphene sheet, and for the cones with $60^{\circ}$ and $120^{\circ}$ disclinations are fitted remarkably well by a straight line, when plotted as a function of $\cos ^{2} \alpha / \sin \alpha$, as shown in Fig. 1. This fact also shows that there is no tip con-

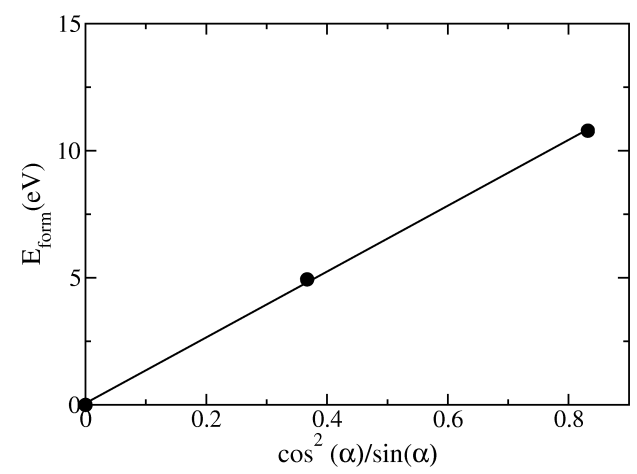

FIG. 1: Formation energy as a function of $\cos ^{2} \alpha / \sin \alpha$ for carbon cones with $60^{\circ}$ and $120^{\circ}$ disclinations and for the graphene sheet.

tribution to the formation energy, i.e. $E_{t i p}=0$, at least for structures with disclination angles up to $120^{\circ}$. This can be explained by the fact that these geometries have the general appearance of truncated cones. Figure 1 also allows us to consider that $R_{c}$ is the same for the cones with $60^{\circ}$ and $120^{\circ}$ disclinations.

Adding the APB energies, we write

$$
E_{\text {form }}=K\left(\frac{\cos ^{2} \alpha}{\sin \alpha}\right) \ln \left(\frac{R}{R_{c}}\right)+E_{A P B}
$$

for the formation energy of BN cones. The constant of proportionality $K$ can be obtained simply by considering that for a $120^{\circ}$ disclination there is no APB, hence $E_{A P B}=0.28$ Having $K$ and the formation energies, we can use the equation above to estimate the APB energies.

\section{FIRST PRINCIPLES RESULTS}

Figure 2 shows three of the most stable structures found in our study. Note that each contains a $60^{\circ}$ disclination, leading to the formation of an APB. We considered two kinds of APB's: one contains a sequence of parallel (N-N or B-B) bonds, as shown in Figs. 2(a) and 2(c), and the other a sequence of zig-zag (N-N or B-B) bonds. These are denoted molecular APB and zig-zag APB, respectively ${ }^{16}$ For cones with $120^{\circ}$ disclinations, we considered structures with a four-membered ring at the apex (without non-BN bonds), as well as structures with two pentagons at the apex, adjacent or not. Disclinations of $180^{\circ}$ and $300^{\circ}$ (each containing an APB) were also addressed. To make contact with experimental results for the synthesis of BN cones from carbon templates, we also considered the possibility of carbon incorporation in $\mathrm{BN}$ cones, leading to the formation of carbon zig-zag [shown in Fig. 2(e)] and molecular APB's. In these structures, every $(\mathrm{B}$ or $\mathrm{N}$ ) atom along the $\mathrm{APB}$ is replaced by a carbon atom. a)

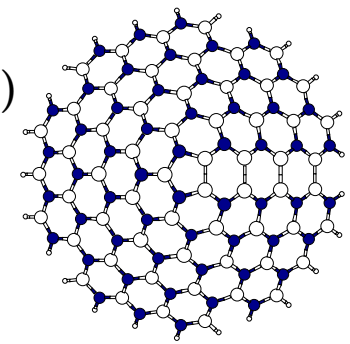

c)

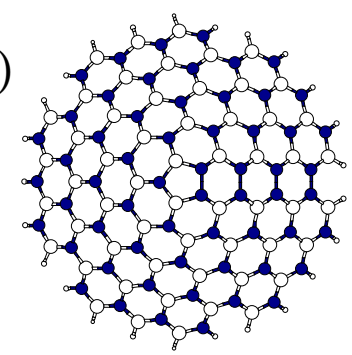

e)

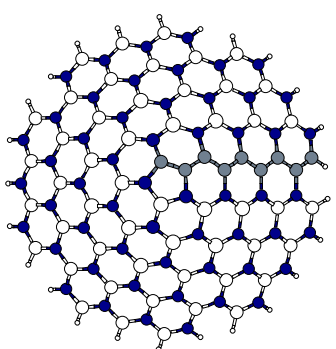

(b)
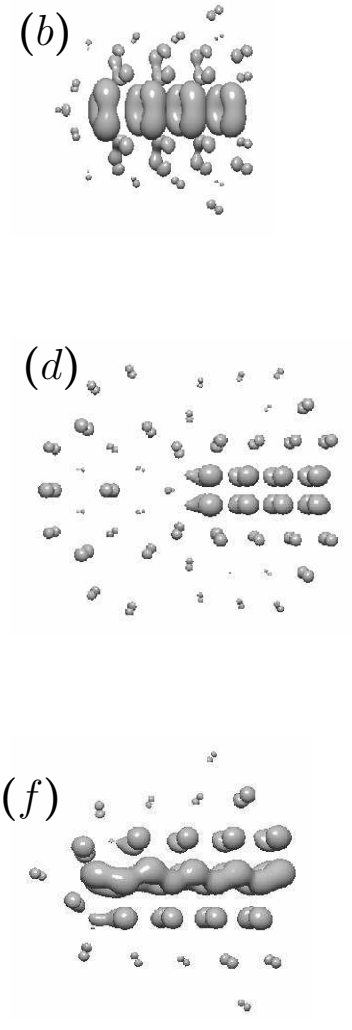

FIG. 2: (Color online) Stable boron nitride nanometer-sized conical structures with $60^{\circ}$ disclinations and an APB (see text). Boron, carbon, and nitrogen atoms are represented by white, gray, and black (blue) circles, respectively. Hydrogen atoms saturate the dangling bonds at the edges. (a) and (b) show a molecular APB of boron and the electron distribution of its empty gap states, respectively. (c) and (d) show the same for a nitrogen molecular APB and its filled gap states. (e) and (f) show the same for a zig-zag APB of carbon and its filled gap states.

Our first principles results for the formation energy of cones with various disclination angles, for B-rich and N-rich environments, are shown in Table I. For the $60^{\circ}-$ disclination cones, the third and fourth columns of Table I show the ratio $E_{A P B} / E_{e l}$, where $E_{e l}=2.96 \mathrm{eV}$, from the continuum model. Note that the highest APB energies are associated with zig-zag configurations, more specifically a zig-zag boron APB in the N-rich environment, and a zig-zag nitrogen APB in the B-rich environment. Geometric aspects may explain these results. In- 
deed, zig-zag APB's introduce larger strains in the structures when compared to molecular APB's, since the parallel arrangement of $\mathrm{B}-\mathrm{B}$ and $\mathrm{N}-\mathrm{N}$ bonds in the latter is more easily accommodated by the $\mathrm{BN}$ matrix of the cone. Table I also shows that for some structures the energetic cost of creating an APB is comparable to the elastic energy, i.e. $E_{A P B} / E_{e l} \sim 1$. For the B-rich environment, these are the molecular APB of boron [shown in Fig. 2(a)] and the zig-zag APB of carbon (with C-B bonds), while for the N-rich environment, the ratio is $\sim 1$ for the molecular APB of nitrogen [shown in Fig. 2(c)] and the zig-zag APB of carbon (with $\mathrm{C}-\mathrm{N}$ bonds)[shown in Fig. 2(e)]. From these observations, we expect that cones with these APB's will be the best candidates to compete energetically with APB-free cones.

Our results for the formation energies, shown in the last two columns of Table I, confirm this expectation. As discussed in Ref. 14, in a condition of chemical equilibrium, the relative stability of the structures is determined by the quantity $E_{\text {form }} / n_{\text {poor }}$, where $n_{\text {poor }}$ is the number of atoms of the species for which the environment is poor $\left[n_{B}\left(n_{N}\right)\right.$ in the N-rich (B-rich) case]. The underlined numbers in Table I indicate the most stable structures. A general trend that can be inferred from the numbers in table I is that the existence of non B-N bonds does not necessarily increase the formation energy. The stability of non-stoichiometric cones depends strongly on the environment. This conclusion is valid for isolated non B-N bonds as well as for the different kinds of APB's.

Concentrating on the structures with $120^{\circ}$ disclinations, we note that, under N-rich conditions, the energy of a cone with two non-adjacent pentagons (containing three $\mathrm{N}-\mathrm{N}$ bonds) is comparable to that of a cone with a four-membered ring at the apex. The same is true if the pentagons are adjacent. The latter is a stoichiometric structure with only one non-BN bond, and its formation energy is independent of the environment.

Concerning the APB's in geometries with $60^{\circ}$ disclinations, we remark again that under the two limiting stoichiometric conditions we considered, $60^{\circ}$ disclinations with $E_{A P B} / E_{e l} \sim 1$ [the molecular APB of nitrogen (in the N-rich case) and the molecular APB of boron (in the B-rich case)] can be more stable than the APB-free structures with $120^{\circ}$ disclinations. These are stable structures, despite being responsible for the existence of gap states 16 (Gap states are absent in the case of cones with a $120^{\circ}$ disclination.)

The electronic charge densities associated with the gap states for the molecular APB's of boron and nitrogen are shown in Figs. 1(b) and 1(d), respectively. The nitrogen-APB states show a substantial degree of delocalization, spreading over the BN matrix of the cone, while the boron-APB states remain strongly localized on the $\mathrm{B}$ atoms at the APB. In a previous study ${ }^{16}$ we examined the electronic levels of these two structures. In the nitrogen case, we found that three fully occupied levels appear in the lower half of the gap, and Fig. 1(d) shows that they have the same nitrogen lone-pair char-
TABLE I: Formation energies (in eV) of stoichiometric and non-stoichiometric cones, for N-rich and B-rich environments. The first column indicates the disclination angle for each structure. Second column indicates either the type of APB or the topological defect at the apex, in each case. For the $120^{\circ}$ and $180^{\circ}$ disclinations, we considered different possibilities for the defect at the apex (indicated by the number of squares and pentagons in each case). The third and fourth columns show the ratio between the the antiphase-boundary $\left(E_{A P B}\right)$ and elastic energies $\left(E_{e l}\right)$ for the $60^{\circ}$ disclinations. The last two columns show the formation energy per $n_{\text {poor }}$, as discussed in the text. Underlined numbers indicate the most stable structures for each environment.

\begin{tabular}{|c|c|c|c|c|c|}
\hline Discl. & Cone & $\begin{array}{c}\frac{E_{A P B}}{E_{e l}} \\
\text { (N-rich) }\end{array}$ & $\begin{array}{c}\frac{E_{A P B}}{E_{e l}} \\
\text { (B-rich) }\end{array}$ & $\begin{array}{c}\frac{E_{\text {form }}}{n(B)} \\
\text { (N-rich) }\end{array}$ & $\begin{array}{c}\frac{E_{\text {form }}}{n(N)} \\
\text { (B-rich) }\end{array}$ \\
\hline $120^{\circ}$ & 1 sq. & & & 0.15 & 0.15 \\
\hline $120^{\circ}$ & 2 pent. & & & 0.17 & 0.30 \\
\hline $120^{\circ}$ & 2 ad. pent. & & & 0.12 & 0.14 \\
\hline $60^{\circ}$ & Zig-N & 2.9 & 7.9 & 0.21 & 0.44 \\
\hline $60^{\circ}$ & Zig-B & 7.7 & 2.7 & 0.43 & 0.20 \\
\hline $60^{\circ}$ & Mol-B & 3.8 & 1.3 & 0.24 & $\underline{0.12}$ \\
\hline $60^{\circ}$ & Mol-N & 1.1 & 3.8 & 0.11 & 0.24 \\
\hline $60^{\circ}$ & Zig-C (C-B) & 4.4 & 1.2 & 0.29 & 0.13 \\
\hline $60^{\circ}$ & Zig-C (C-N) & 0.6 & 3.8 & $\underline{0.09}$ & 0.26 \\
\hline $60^{\circ}$ & Mol-C (C-B) & 7.1 & 2.1 & 0.43 & 0.18 \\
\hline $60^{\circ}$ & Mol-C (C-N) & 1.4 & 6.0 & 0.14 & 0.37 \\
\hline $180^{\circ}$ & 3 pents & & & 0.48 & 0.85 \\
\hline $180^{\circ}$ & 1 pent. +1 sq. & & & 0.42 & 0.66 \\
\hline $300^{\circ}$ & Mol-N & & & 1.44 & 1.61 \\
\hline
\end{tabular}

acter that dominates the states at the top of the valence band of the cone. This leads to a strong mixing between the APB states and the valence-band states in the bulk of the cone, this being the reason for the delocalized character of the nitrogen-APB states. In the case of boron, the states shown in Fig. 1(b) are empty levels near the bottom of the conduction band. They have a more metallic character, differing from the states at the bottom of the conduction band. Mixing is absent in this case, leading to the localized distribution seen in Fig. 1(b).

Table I also shows that cones with zig-zag APB's have higher formation energies, and the same occurs for structures with large disclination angles. The result is expected in the case of the $180^{\circ}$ and $300^{\circ}$ disclinations, since besides the cost of forming an APB, the elastic energy is also large. However, we note that these metastable structures have already been found experimentally ${ }^{8.18}$

Regarding carbon-doped cones, we find that incorporation of carbon at the APB leads to highly stable structures. In this case, for both kinds of APB's the most stable structures are those where the APB is connected to the $\mathrm{BN}$ matrix by $\mathrm{C}-\mathrm{N}$ (C-B) bonds in the N-rich (B-rich) environment. Actually, the most stable of all structures in our study is the C-N connected carbon zig-zag APB, in the N-rich environment, shown in Fig. 1(e). The elec- 
tronic charge density of the gap states associated with this structure is shown in Fig. 1(f). The states are predominantly localized in the antiphase boundary, strongly resembling the states characteristic of the graphite $\pi$ band. This wavefunction localization leads also to a considerable spin splitting at the Fermi level. For the neutral charge state, the energy difference between the highest-occupied molecular orbital (which has majority spin) and the lowest-unoccupied one (which has minority spin) is $\sim 0.5 \mathrm{eV}$. This spin splitting appears only for structures with an odd number of electrons, a result that raises the interesting question about what may happen in the limit of cones with larger sizes. Furthermore, we have also identified a tendency for carbon atoms to segregate at the APB. In our calculations, a single pair of carbons atoms is found to be more stable (by $\sim 0.07$ $0.15 \mathrm{eV} /$ atom) on the APB than in the bulk of a BN cone. This may indicate a tendency for the formation of these carbon-modified APB's, during the synthesis of $\mathrm{BN}$ conical nanostructures by substitution on a carbon template.

Boron nitride cones can also be obtained by a substitution reaction ${ }^{18}$ on a carbon template in which $\mathrm{B}_{2} \mathrm{O}_{3}$ and gaseous $\mathrm{N}_{2}$ are used as sources of boron and nitrogen, respectively. To check the stability trends in this case, we consider that $\mathrm{BN}$ cones and $\mathrm{CO}_{2}$ are the byproducts of this reaction. The nitrogen and boron chemical potentials are then obtained in terms of the total energies of the species involved 29 With this procedure, the value we obtained for the chemical potential difference, $\mu_{N}-\mu_{B}=-189.936 \mathrm{eV}$, is very close to the value of $-190.126 \mathrm{eV}$, obtained for an N-rich environment under the constraint in Eq. 1. Therefore, the stability trends shown in table I still hold in this case. We also checked the robustness of our results with respect to changes in the boundary conditions, which could happen, for instance, if the cones were interacting with different surfaces. This was done by imposing a constant shift of $\pm 0.5 \mathrm{eV}$ on all hydrogen chemical potentials. The ordering of the quantities $E_{\text {form }} / n_{\text {poor }}$ in table I remains unaltered, with relative shifts of less than $0.02 \mathrm{eV}$. Still to confirm the validity of our methodology, we mention that similar approaches have been employed in the literature in the determination of formation energies of defects in cubic boron nitride $\stackrel{24.25 .26}{ }$ in boron nitride nanotubes 27 and in the investigation of possible structures of boron nitride fullerenes ${ }^{14}$ In the latter work, the stability trends obtained by using the solid phases of boron and nitrogen together with the constraint in Eq. 1 remained largely unchanged when the authors considered the gas phase limit of atomic reservoirs.

\section{REMARKS ON SIZE SCALING AND CONCLUSION}

Finally, we discuss the relevance of our results in face of the expected scaling trends. As the size of a cone increases, the APB energy scales linearly with the slant height $R$ (see Eq. 5), i.e. with the square root of the total number of atoms in the structure, while the elastic energy scales sub-linearly with $R$, as shown in Eq. [5] Hence, structures without APB's become energetically favored for sufficiently larger sizes. However, cones of the size we consider in this work may represent the seeds present in the initial steps of the nucleation process that generates larger cones or other nanostructures. Not only the energetics, but also kinetics play an important role in this process, and to the extent that we may expect low energy configurations to be favored for the seeds, cones with certain types of APB's may be good candidates, and kinetics may determine the formation of larger size structures containing APB's.

To summarize, we used first-principles calculations to address the question of how stable are antiphase boundaries in boron nitride nanometer-sized conical nanostructures. Our results indicate that $60^{\circ}$ disclinations with an APB can be more stable than APB-free $120^{\circ}$ disclinations. We show that the formation energies of the stable APB's are comparable to the disclination elastic energy, and that the dependence of the latter on the disclination angle is very well described by a continuum model. Also, we observe that carbon incorporation at the APB leads to highly stable structures, of which the most stable presents a large spin splitting $(\sim 0.5 \mathrm{eV})$ at the Fermi level.

\section{Acknowledgments}

We acknowledge support from the Brazilian agencies CNPq, FAPEMIG, and Instituto do Milênio em Nanociências-MCT.
* Electronic address: chacham@fisica.ufmg.br

1 S. Iijima, Nature (London) 354, 56 (1991).

2 N. G. Chopra, R. J. Luyken, K. Cherrey, V. H. Crespi, M. L. Cohen, S. G. Louie, and A. Zettl, Science 260, 966 (1995).

3 Z. Weng-Sieg, K. Cherrey, N. G. Chopra, X. Blase, Y. Miyamoto, A. Rubio, M. L. Cohen, S. G. Louie, A. Zettl and R. Gronsky, Phys. Rev. B 51, 11229 (1995).
4 A. Loiseau, F. Willaime, N. Demoncy, G. Hug, and H. Pascard Phys. Rev. Lett. 76. 4737 (1996).

${ }^{5}$ H. W. Kroto, J. R. Heath, S. C. O'Brien, R. F. Curl, and R. E. Smalley, Nature (London) 318, 162 (1985).

6 D. Golberg, Y. Bando, K. Kurashima, and T. Sasaki Appl. Phys. Lett. 72, 2108 (1998).

7 A. Krishnan, E. Dujardin, M. M. J. Treacy, J. Hugdahl, S. Lynum, and T. W. Ebbesen. Nature 388451 (1997) 
${ }^{8}$ L. Bourgeois, Y. Bando, W. Q. Han and T. Sato. Phys. Rev. B 61, 7686 (2000).

9 P. Zhang and V. H. Crespi. Phys. Rev. B 6211050 (2000)

10 T. Dumitrica, H. F. Bettinger, G. Scuseria and B. I. Yakobson. Phys. Rev. B 68085412 (2003)

11 For a definition and examples of disclinations in BN nanostructures see reference ${ }^{16}$

12 Simone S. Alexandre, Mário S. C. Mazzoni, and Hélio Chacham Appl. Phys. Lett. 75, 61 (1999).

${ }^{13}$ F. Jensen and H. Toftlund. Chem. Phys. Lett. 201, 89 (1993).

14 Simone S. Alexandre, Hélio Chacham, R. W. Nunes. Phys. Rev. B 63045402 (2001).

15 P. W. Fowler, K. M. Rogers, G. Seifert, M. Terrones, and H. Terrones, Chem. Phys. Lett. 299, 359 (1999).

16 S. Azevedo, Mário S. C. Mazzoni, H. Chacham, R. W. Nunes. Appl. Phys. Lett. 822323 (2003)

17 A. Loiseau, F. Willaime, N. Demoncy, N. Schromchenko, G. Hug, C. Colliex and H. Pascard Carbon. 36. 743 (1998).

18 D. Golberg, Y. Bando, L. Bourgeois, K. Kurashima, and T. Sato, Appl. Phys. Lett 77, 1979 (2000).

19 W. Kohn, L. J. Sham. Phys. Rev. 140, A1133 (1965).

20 D. Sanchez-Portal, P. Ordejon, E. Artacho and J. M. Soler. Int. Journ. of Quant. Chem. 65, 453 (1997).

21 N. Troullier and J. L. Martins. Phys. Rev. B. 43, 1993 (1991).

${ }^{22}$ L. Kleinman and D. M. Bylander. Phys. Rev. Lett. 48,
$1425(1982)$.

23 J. P. Perdew, K. Burke, M. Ernzerhof. Phys. Rev. Lett. 77 3865 (1996)

${ }^{24}$ W. Orellana and H. Chacham. Appl. Phys. Lett. 74, 2984 (1999)

25 W. Orellana and H. Chacham. Phys. Rev. B. 62, 10135 (2000)

26 W. Orellana and H. Chacham. Phys. Rev. B. 63, 125205 (2001)

27 T. M. Schmidt, R. J. Baierle, P. Piquini, and A. Fazzio. Phys. Rev. B. 67, 113407 (2003).

28 There is a very small difference in slant height between our $60^{\circ}$ - and $120^{\circ}$-disclination structures, which leads to a negligible change in the logarithmic term in Eq. 5 between these two types of structures. This has been neglected in our determination of $K$.

29 The substitution reaction described allows us to write the nitrogen and boron chemical potentials as $\mu_{N}=\frac{1}{2} E\left(N_{2}\right)$ and $\mu_{B}=\frac{1}{2}\left[E\left(B_{2} O_{3}\right)+\frac{3}{2} E(C)-\frac{3}{2} E\left(C O_{2}\right)\right]$, where $\mathrm{E}\left(\mathrm{N}_{2}\right)$ and $\mathrm{E}\left(\mathrm{CO}_{2}\right)$ are the total energies of the nitrogen and carbon dioxide molecules, respectively, $\mathrm{E}\left(\mathrm{B}_{2} \mathrm{O}_{3}\right)$ is the total energy of a planar hexagonal $\mathrm{B}_{2} \mathrm{O}_{3}$ structure and $\mathrm{E}(\mathrm{C})$ is the energy per atom in graphene. All these quantities are obtained by means of first-principles calcultions and the resulting values of $\mu_{N}$ and $\mu_{B}$ are $-270.151 \mathrm{eV}$ and -80.214 $\mathrm{eV}$, respectively. 